\title{
Estudio técnico-económico de la producción de leche de cabra en polvo
}

\author{
LOURDES CRESPo-ZAFrA ${ }^{1}$ \\ AMAURY PÉREZ-SÁNCHEZ ${ }^{1}$ \\ LISANDRO RAMOS-LÓPEZ ${ }^{1}$ \\ EDUARDO GARCÍA-NOA ${ }^{2}$ \\ LUISA MATOS-MOSQUEDA ${ }^{1}$
}

\section{Resumen}

En el presente trabajo se determinó la rentabilidad económica de una planta de producción de leche de cabra en polvo en Cuba, con una capacidad de 106 ton/año (508 kg/lote) mediante el empleo del simulador de procesos SuperPro Designer ${ }^{\circledR}$ versión 8.5. También se evaluó la influencia de la adición de un segundo secador por aspersión (Variante) sobre la rentabilidad global del proceso productivo. Por último, se realizó un estudio de sensibilidad para obtener correlaciones estadísticas que relacionen tres variables iniciales con tres indicadores económicos: 1) Valor Actual Neto (VAN); 2) Tasa Interna de Retorno (TIR); y 3) Periodo de Recuperación de la Inversión (PRI). Se requiere una inversión total de \$2 772000 pesos cubanos (PC) para erigir la planta, se obtienen ganancias totales anuales de PC \$1.173.000, mientras que el VAN, TIR y PRI tuvieron valores de PC \$3.842.000; 33,83 \% y 2,70 años, respectivamente, calificando el proceso de económicamente rentable. La aplicación de la Variante reduce la rentabilidad económica global del proceso productivo.

Palabras Clave: Estudio de sensibilidad; Leche de cabra en polvo; Producción; Rentabilidad; Super Pro Designer@.

\section{Techno-economic study of the goat milk powder production}

\section{Abstract}

In the present work, the economic profitability of a powder goat milk production plant in Cuba, with a production capacity of 106 tons/year (508 kg/batch) was determined by using the SuperPro Designer® process simulator version

\footnotetext{
Universidad de Camagüey. Camagüey, Cuba.

Universidad Tecnológica de La Habana. La Habana. Cuba.
}

Autor de correspondencia: Pérez Sánchez, A. (Amaury): Departamento de Ingeniería Química, Facultad de Ciencias Aplicadas, Universidad de Camagüey, Carretera Circunvalación Norte, Km. 51⁄2, e/ Camino Viejo de Nuevitas y Ave. Ignacio Agramonte, CP 74650, Camagüey, Cuba. Teléfono: +53 32286385 Email: amaury.psanchez@reduc.edu.cu,
Historia del artículo:

Artículo recibido: 10-VI-2019/ Aprobado: 15-I-2020

Disponible online: 15 de enero de 2020

Discusión abierta hasta septiembre de 2021 
8.5. The influence of the addition of a second spray dryer (Variant) on the overall profitability of the production process was also evaluated. Finally, a sensitivity study was conducted in order to obtain statistical correlations that relate three initial variables with three economic indicators: 1) Net Present Value (NPV); 2) Internal Rate of Return (IRR); and 3) Payback Period (PP). It's required a total investment of \$2,772,000 Cuban pesos (CP) to erect the plant, total annual profits of CP \$1,173,000 are obtained, while the NPV, IRR and PP had values of CP \$3,842,000; $33.83 \%$ and 2.70 years, respectively, qualifying the process as economically profitable. The application of the Variant reduces the overall economic profitability of the production process.

Keywords: Sensitivity study; Goat milk powder; Production; Profitability; SuperPro Designer®.

\section{Estudo técnico-econômico da produção de leite em pó de cabra}

\section{Resumo}

Neste trabalho foi determinada a rentabilidade económica de uma produção de leite em pó de cabra em Cuba, com uma capacidade de 106 toneladas / ano (508 kg / lote), utilizando o simulador processo Designer SuperPro® versão 8.5 . A influência da adição de um segundo spray dryer (Variant) na rentabilidade global do processo de produção também foi avaliada. Por fim, foi realizado um estudo de sensibilidade para obter correlações estatísticas que relacionam três variáveis iniciais com três indicadores econômicos: 1) Valor Presente Líquido (VPL); 2) Taxa Interna de Retorno (TIR); e 3) Período de Recuperação do Investimento (PRI). um investimento total de US \$2,772 milhões de pesos cubanos (PC) é necessário para erguer a planta, rendimento anual total de US \$1.173.000 são obtidas PC, enquanto o VPL, TIR valores e PC do PRI foram de US \$ 3.842.000; 33,83\% e 2,70 anos, respectivamente, qualificando o processo economicamente rentável. A aplicação da Variant reduz a rentabilidade econômica geral do processo de produção.

Palavras chave: Estudo de sensibilidade; Leite em pó de cabra; Produção; Lucratividade; Super Pro Designer@

\section{Introducción}

Los productos lácteos han sido reconocidos como una parte importante de la dieta humana, tanto en países desarrollados como en vías de desarrollo. La leche de cabra (Capra hircus) contiene agua, proteínas, grasas, azúcares, minerales y vitaminas, las cuales son esenciales para el mantenimiento de una buena salud (Pal et al., 2017). Su calidad está enlazada con singularidades históricas y culturales directamente a través de las cadenas de producción, mercadotecnia y consumo (Yangilar, 2013). En años recientes la leche de cabra y sus productos están recibiendo gran atención en varios países del mundo. La leche de cabra fluida y sus productos procesados son útiles como alimentos funcionales para el mantenimiento de la nutrición y salud de personas jóvenes y longevas, y especialmente para aquellas que padecen de alergia a la leche de vaca. La leche de cabra puede ser empleada para preparar una gran variedad de subproductos lácteos fundamentalmente quesos (Park, 1990; Gomes \& Malcata, 1998; Seifu et al., 2004;
Guo et al., 2004; Mukdsi et al., 2013); yogurt (Domagala, 2009; Vargas et al., 2008; Kücükcetin et al., 2011); helados (Correia et al., 2008; Pandya \& Ghodke, 2007; Ranadheera et al., 2013), leche en polvo (Prosser et al., 2008; Pal, 2014; Reddy et al., 2014) y otros productos incluyendo leche condensada y mantequilla (Pandya \& Ghodke, 2007; Rodriguez et al., 2003).

La leche en polvo se prepara a partir de la remoción del agua contenida en la leche líquida (Pal et al., 2017). Presenta mejores cualidades para el mantenimiento de su calidad, demanda de menos espacio de almacenamiento y requiere menores costos de transportación (Pal, 2014).

El proceso de obtención de leche de cabra en polvo ha sido estudiado por diferentes autores. En este sentido, Reddy y colaboradores (Reddy et al., 2014) optimizaron las condiciones de procesamiento para la fabricación de leche de cabra en polvo, tomando en cuenta como variables independientes la concentración de sólidos de la leche (35, 40 y 45\%) y la temperatura del 
aire de entrada (160, 170 y $180 \stackrel{\circ}{\circ}$ ), obteniéndose una composición aproximada de humedad, grasas, proteínas, carbohidratos, cenizas y acidez de 4,08\%, 26,85\%, $25,48 \%, 36,99 \%, 6,60 \%$ y $0,14 \%$ respectivamente.

También (Altieri et al., 2008) llevaron a cabo estudios para optimizar el proceso de secado por atomización de leche de cabra concentrada, utilizando tres valores de temperatura de entrada $(120,150$ y $180 \stackrel{\circ}{\circ})$, un flujo de alimentación de $0,5 \mathrm{dm}^{3} / \mathrm{h}$ y una humedad relativa del aire de salida en el rango de 10 - $40 \%$. En este caso la leche cruda fue concentrada utilizando un evaporador de baja presión hasta alcanzar una concentración de materia seca del $23 \%$.

Por otro lado, (Ravula et al., 2018) evaluaron sensorialmente la leche de cabra en polvo tanto secada por atomización como aglomerada. La caracterización se realizó tomando en cuenta 4 parámetros: color y apariencia, sabor, consistencia y aceptabilidad total, utilizando una escala hedonista de nueve puntos para puntualizar las diferencias existentes entre las muestras obtenidas mediante secado por atomización, y las aglomeradas. La aceptabilidad global de las muestras secadas por atomización fueron casi todas similares en comparación con la de la leche de vaca en polvo, mientras que la leche aglomerada fue distinta de esta.

El SuperPro Designer ${ }^{\circledR}$ es un simulador profesional de proceso el cual facilita la modelación, evaluación y optimización de procesos integrados en un amplio rango de industrias tales como la farmacéutica, alimentaria, tratamiento de agua y aguas residuales, y procesos que incluyen componentes biológicos. Contiene diferentes herramientas útiles de procesos y operaciones químicas tales como la realización de balances de masa y energía, extensa base de datos de componentes químicos y mezclas, dimensionamiento de equipos, cálculos económicos detallados, caracterización de emisiones y corrientes residuales y de desecho, entre otras funciones (Auli et al., 2013). Su uso se ha extendido en los últimos años para realizar estudios de rentabilidad económica en una gran variedad de industrias y procesos químicos y biotecnológicos (Qureshi et al., 2013; Lam et al., 2014).

En el presente trabajo se realiza la simulación del proceso de producción de leche de cabra en polvo mediante el simulador SuperPro Designer ${ }^{\circledR}$, con el fin de determinar su parámetros técnico-económicos más importantes y determinar la rentabilidad del proceso productivo. También se realiza un análisis de sensibilidad para evaluar la influencia de tres variables de entrada (costo de adquisición de la leche fresca; precio de venta de la leche en polvo y capacidad de producción) sobre los indicadores Valor Actual Neto (VAN), Tasa Interna de Retorno (TIR) y Periodo de Recuperación de la Inversión (PRI), mientras que por último se propone una alternativa de producción (Variante) consistente en la introducción en el proceso productivo de un segundo secador por atomización para reducir el tiempo de secado, evaluándose también el impacto económico de esta propuesta y su posterior comparación con los resultados obtenidos en el Caso Base.

\section{Materiales y métodos}

\section{Composición de la leche de cabra}

Para efectuar la simulación se utilizó la siguiente composición química para la leche de cabra, según (Fox \& McSweeney, 1998):

TABLA 1. COMPOSICIÓN QUÍMICA DE LA LECHEDE CABRA UTILIZADA EN LA SIMULACIÓN

\begin{tabular}{l|c}
\hline Composición & Valor (\%) \\
\hline Sólidos totales & 12,3 \\
\hline Grasas & 4,5 \\
\hline Proteínas & 2,9 \\
\hline Lactosa & 4,1 \\
\hline Cenizas & 0,8 \\
\hline Impurezas & 1,0 \\
\hline Agua & 74,4 \\
\hline
\end{tabular}

Descripción del proceso de producción de leche de calbra en polvo (Caso Base)

En la Figura 1 se presenta el diagrama de flujo del proceso de producción (Caso Base) obtenido mediante el simulador SuperPro Designer ${ }^{\circledR}$. Se vierten 1.977,6 $\mathrm{kg} /$ lote de leche de cabra fresca en un tanque de $4 \mathrm{~m}^{3}$ de volumen, la cual es bombeada a continuación hacia un filtro colador para remover partículas extrañas que puedan existir en suspensión. A la salida del filtro, la leche transita por un intercambiador de calor (enfriador) donde se reduce su temperatura hasta $4{ }^{\circ} \mathrm{C}$ utilizando glicol como medio de enfriamiento. La leche fría a $4{ }^{\circ} \mathrm{C}$ es almacenada en un tanque isotérmico de $4 \mathrm{~m}^{3}$ de ca- 
pacidad. Una vez enfriada, la leche es pasteurizada a 72 ${ }^{\circ} \mathrm{C}$, y luego enfriada hasta $10 \stackrel{\circ}{\circ} \mathrm{C}$ en el mismo equipo, el cual emplea vapor de agua y agua fría como agentes de calentamiento y enfriamiento, respectivamente. La leche pasteurizada a $10^{\circ} \mathrm{C}$ de temperatura es almacenada en un tanque isotérmico de $3,5 \mathrm{~m}^{3}$ de capacidad, desde donde se envía por medio de una bomba peristáltica hacia un intercambiador de calor de tubo en tubo para incrementarle su temperatura hasta $60 \stackrel{\circ}{ } \mathrm{C}$, para entonces ser enviada de forma continua hacia el secador por atomización a un caudal de alimentación de $25 \mathrm{~L} / \mathrm{h}$. En el secador se obtiene la leche en polvo a una temperatura de $80 \stackrel{\circ}{\circ}$ y una humedad de 5,7\%. Por último, la leche en polvo es envasada en bolsas de nailon con una capacidad de almacenamiento de $600 \mathrm{~g}$ cada una, utilizando para ello una llenadora de bolsas con una capacidad de llenado de 36 bolsas por hora.

Aspectos tomados en cuenta para efectuar la simulación

Se toma una capacidad de procesamiento de $1.977,6 \mathrm{~kg}$ de leche de cabra fresca por lote, con un período de construcción de la planta de 18 meses y 3 meses para efectuar la arrancada y puesta en marcha. Todos los valores y resultados tanto económicos como monetarios se expresan en pesos cubanos (PC). La planta operará 11 meses al año, con 1 mes disponible para efectuar labores de mantenimiento de equipos y sistemas auxiliares, mientras que se considera que tendrá un tiempo de vida de 20 años. Se asume un 11\% de interés para determinar el VAN, un $32 \%$ de impuestos por concepto de ingresos, se gastan PC \$5000 al año por concepto de publicidad y ventas, y que la planta operará al $80 \%$ de su capacidad total durante los cinco primeros años, para luego operar al $90 \%$ por el resto del tiempo de vida. Se supone que no existe rechazo de producto por no cumplir con los requisitos de calidad, que el costo de arrancada y validación se toma como un 5\% del capital fijo directo, que el costo relacionado con el funcionamiento del laboratorio se toma como un 15\% del costo total de mano de obra, y que no existe gasto económico debido a procesos de investigación y desarrollo (I+D) en la planta. Se estima la contribución de cada componente sobre la inversión de capital fijo basados en el rango sugerido por (Peters et al., 2003). Se toma además un salario de PC $\$ 1,36 / \mathrm{h}$ para operarios y personal de mantenimiento, y de PC $\$ 2,00 /$ h para supervisores y personal de dirección, mientras que el costo de adquisición tanto de la leche fresca de cabra como de las bolsas de nailon vacías se toma en PC \$5,00/Ly \$ PC 0,10/bolsa, respectivamente. La planta emplea todos los servicios auxiliares comúnmente consumidos en una planta de este tipo, esto es, agua de enfriamiento, electricidad, vapor de agua y aire comprimido (Tabla 2). Se asumió que el costo de tratamiento de aguas residuales (principalmente aguas residuales provenientes de las operaciones de limpieza y desinfección de equipamiento, accesorios y áreas) es de $30 \%$ de los costos de operación, y se especificó un costo de la electricidad de $\$ 1,20 / \mathrm{kWh}$. Por último, el costo de venta de la bolsa de leche de cabra en polvo de 600 g se toma igual a PC \$18,00.

\section{Figura 1. Diagrama de flujo del proceso de producción de leche de cabra en polvo (Caso Base)}

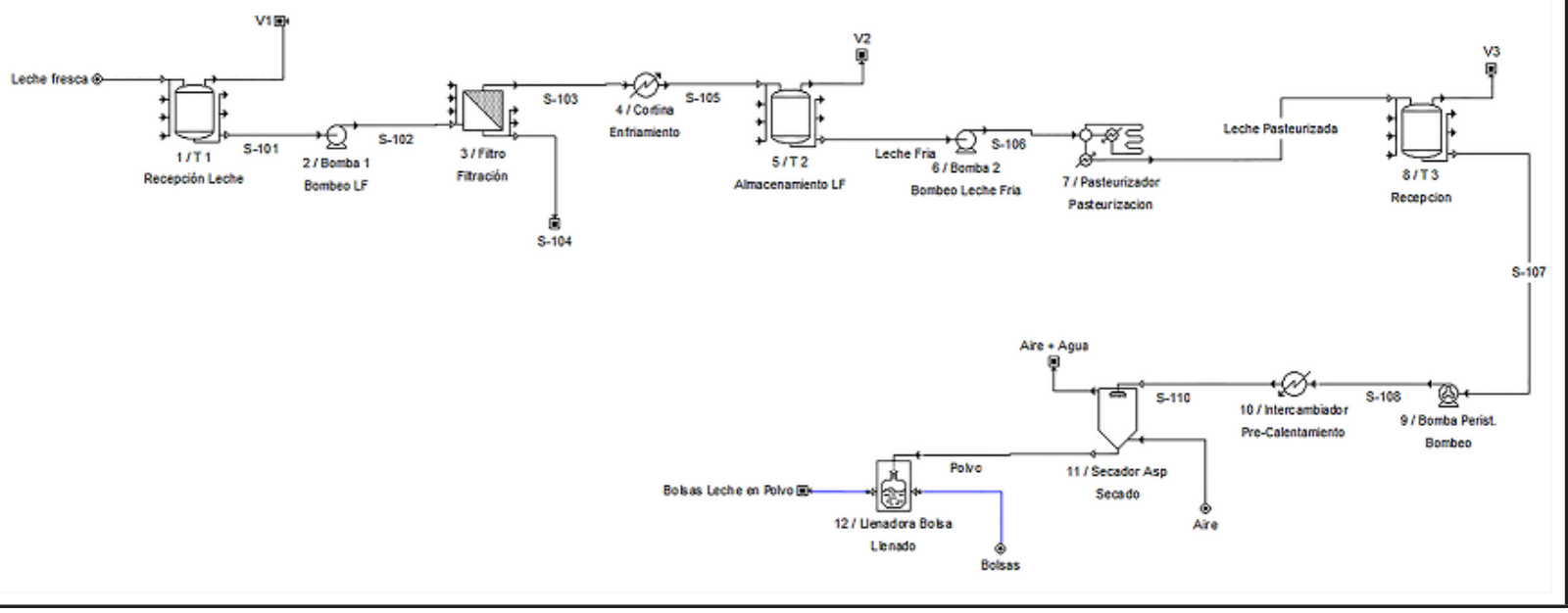


TABLA 2. SERVICIOS AUXILIARES CONSUMIDOS POR LA PLANTA DE PRODUCCIÓN, Y SU COSTO UNITARIO

\begin{tabular}{l|c} 
Servicio auxiliar & Costo unitario (PC \$/MT) \\
\hline Agua fría & 0,40 \\
\hline Agua de enfriamiento & 0,05 \\
\hline Vapor de agua & 3,50 \\
\hline
\end{tabular}

\section{Costo del equipamiento}

La siguiente tabla (Tabla 3) muestra el costo del equipamiento utilizado en el proceso productivo (Peters et al., 2003; Towler \& Sinnott, 2008, Perry \& Green, 2008).

\begin{tabular}{|c|c|c|}
\hline Equipo & Descripción & $\begin{array}{l}\text { Costo } \\
\text { (PC \$) }\end{array}$ \\
\hline $\begin{array}{l}\text { Tanque de recepción } \\
\text { de leche }\end{array}$ & Volumen: $4000 \mathrm{~L}$ & 12.000 \\
\hline Bomba centrífuga 1 & Potencia: 5 kW & 173 \\
\hline Filtro colador & & 57 \\
\hline $\begin{array}{l}\text { Enfriador de leche } \\
\text { fresca }\end{array}$ & $\begin{array}{l}\text { Área de transferencia } \\
\text { de calor: } 80 \mathrm{~m}^{2}\end{array}$ & 549 \\
\hline $\begin{array}{c}\text { Tanque de } \\
\text { almacenamiento de } \\
\text { leche fría }\end{array}$ & Volumen: $4000 \mathrm{~L}$ & 12.000 \\
\hline Bomba centrífuga 2 & Potencia: $5 \mathrm{~kW}$ & 173 \\
\hline Pasteurizador & Capacidad: 2000 L/h & 1.768 \\
\hline $\begin{array}{c}\text { Tanque } \\
\text { almacenamiento leche } \\
\text { pasteurizada }\end{array}$ & Capacidad: $3500 \mathrm{~L}$ & 12.000 \\
\hline Bomba peristáltica & Potencia: 1,5 kW & 1.500 \\
\hline Precalentador & $\begin{array}{c}\text { Área de transferencia } \\
\text { de calor: } 50 \mathrm{~m}^{2}\end{array}$ & 600 \\
\hline $\begin{array}{l}\text { Secador por } \\
\text { atomización }\end{array}$ & $\begin{array}{c}\text { Diámetro: 1,60 m } \\
\text { Altura: } 3,50 \mathrm{~m}\end{array}$ & 253.400 \\
\hline Llenadora de bolsas & $\begin{array}{l}\text { Capacidad: } 36 \\
\text { bolsas } / \mathrm{h}\end{array}$ & 15.000 \\
\hline
\end{tabular}

\section{Estudio de sensibilidad}

Se procederá a evaluar la influencia de 3 variables de entrada o iniciales, las cuales son 1) costo de adquisición de la leche de cabra fresca; 2 ) precio de venta de la leche de cabra en polvo; y 3) capacidad de producción de leche de cabra en polvo por lote, sobre tres indicadores de salida: Valor Actual Neto (VAN), Tasa Interna de Retorno (TIR) y Período de Recuperación de la Inversión
(PRI). Las tres variables de entrada consideradas fueron especificadas para variar dentro de un porcentaje determinado, con el fin de tomar en cuenta fluctuaciones esperadas de sus valores en el futuro (Tabla 4).

TABLA 4. VALORES QUE DEBERÁN PRESENTAR CADA VARIABLE DE ENTRADA CONSIDERANDO EL PORCENTAJE DE VARIACIÓN SELECCIONADO, CON EL FIN DE EFECTUAR EL ESTUDIO DE SENSIBILIDAD

\begin{tabular}{|l|c|c|c|}
\hline \multicolumn{1}{|c|}{ Parámetro } & $\begin{array}{c}\text { Valor } \\
\text { Inicial }\end{array}$ & $\begin{array}{c}\text { Valor } \\
\text { mínimo } \\
\mathbf{( - 3 0} \%)\end{array}$ & $\begin{array}{c}\text { Valor } \\
\text { máximo } \\
\mathbf{( + 3 0 \% )}\end{array}$ \\
\hline $\begin{array}{l}\text { Costo de adquisición } \\
\text { leche fresca (PC \$/L) }\end{array}$ & 5,0 & 3,5 & 6,5 \\
\hline $\begin{array}{l}\text { Precio de venta leche } \\
\text { en polvo (PC \$/bolsa) }\end{array}$ & 18,0 & 12,6 & 23,4 \\
\hline $\begin{array}{l}\text { Capacidad de } \\
\text { producción (kg/lote) }\end{array}$ & 508 & 355,6 & 660,4 \\
\hline
\end{tabular}

\begin{tabular}{|c|c|c|c|}
\hline Corrida & $\begin{array}{c}\text { Costo leche } \\
\text { fresca } \\
\text { [PC \$/L] }\end{array}$ & $\begin{array}{l}\text { Precio de venta } \\
\text { leche en polvo } \\
\text { [PC } \$ / \text { bolsa] }\end{array}$ & $\begin{array}{l}\text { Capacidad de } \\
\text { producción } \\
\text { [kg/lote] }\end{array}$ \\
\hline 1 & 6,5 & 12,6 & 355,6 \\
\hline 2 & 5,0 & 12,6 & 355,6 \\
\hline 3 & 3,5 & 23,4 & 660,4 \\
\hline 4 & 3,5 & 12,6 & 355,6 \\
\hline 5 & 3,5 & 23,4 & 355,6 \\
\hline 6 & 6,5 & 23,4 & 660,4 \\
\hline 7 & 6,5 & 12,6 & 355,6 \\
\hline 8 & 6,5 & 23,4 & 355,6 \\
\hline 9 & 6,5 & 12,6 & 660,4 \\
\hline 10 & 3,5 & 12,6 & 660,4 \\
\hline 11 & 5,0 & 23,4 & 660,4 \\
\hline 12 & 3,5 & 23,4 & 508,0 \\
\hline
\end{tabular}

Con el objetivo de generar el estudio de sensibilidad se elaboró un diseño de experimentos del tipo Superficie de Respuesta a través del paquete estadístico Statgraphics Centurion, obteniendo 27 corridas de forma inicial, las cuales fueron posteriormente optimizadas mediante el empleo de la herramienta "D-Optimality" contenida en el propio paquete estadístico, con el propósito de reducir el diseño de experimentos y seleccionar aquellas corridas que presentan mayor influencia 
estadística en el estudio de sensibilidad, obteniendo finalmente 12 corridas experimentales (Tabla 5).

\section{Evaluación de la correlación estadística} existente entre las variables de entrada y los indicadores económicos de salida

Una vez efectuado el estudio de sensibilidad, se procedió a determinar la correlación estadística existente entre las 3 variables de entrada consideradas y los 3 indicadores económicos de salida evaluados, con el objetivo de obtener correlaciones o ecuaciones que describan, de forma cuantitativa, la relación estadística existente entre estas variables. Lo anterior se efectuó mediante el empleo de la opción "Multiple Regression" contenida en el paquete estadístico Statgraphics Centurion.

Por último, se determinó la corrida (o escenario) con el resultado económico más positivo con respecto a los valores de VAN, TIR y PRI obtenidos, asi como también aquella con el más negativo.

\section{Propuesta de alternativa de producción (Variante)}

Se consideró adicionar un segundo secador por atomización al proceso productivo (Figura 2), con el fin de acortar el tiempo de procesamiento de un lote y evaluar así la influencia de este cambio en el posible incremento de la rentabilidad global de la planta de producción. La introducción de un equipo de este tipo en el proceso productivo también conlleva la inclusión de una bomba peristáltica y un precalentador adicional, los cuales tendrán un costo de adquisición similar al empleado en el Caso Base para estos equipos, esto es, PC \$ 1.500 y PC \$ 600 respectivamente. El secador por atomización también tendrá un costo de adquisición semejante con respecto al utilizado en el Caso Base, es decir, PC \$ 253 400. Para la simulación de la Variante se tomaron en cuenta todos los aspectos y cuestiones asumidos con anterioridad para llevar a cabo la simulación del Caso Base.

\section{Resultados y discusión}

Principales indicadores técnico económicos obtenidos durante la simulación del Caso Base

La Tabla 6 muestra los resultados de los principales indicadores técnico-económicos obtenidos durante la simulación del proceso de producción de leche de cabra en polvo (Caso Base) en el SuperPro Designer ${ }^{\circledR}$.

Figura 2. Diagrama de flujo de proceso de producción de leche de cabra en polvo utilizando dos secadores por atomización (Variante)

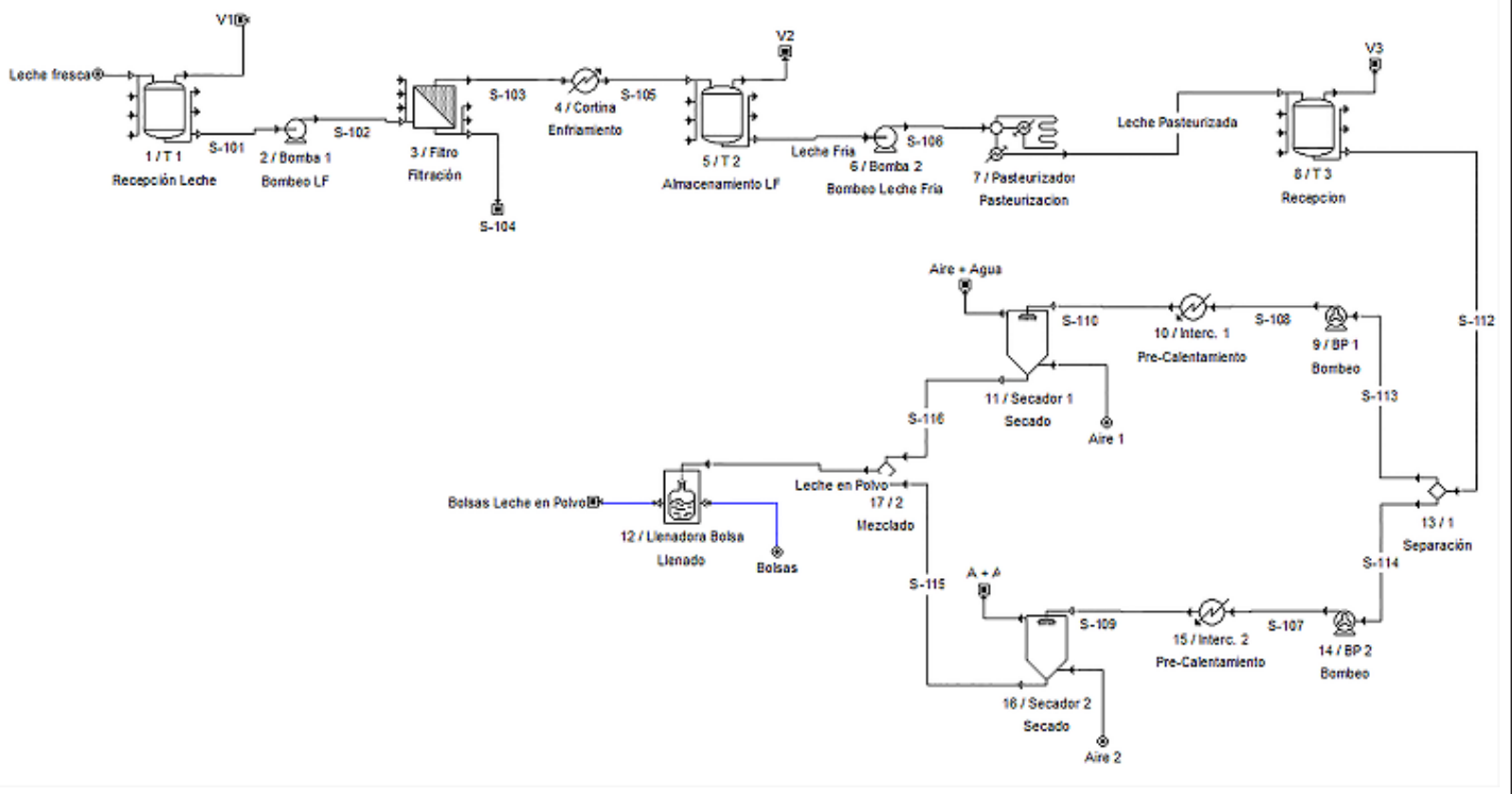


TABLA 6. PRINCIPALES INDICADORES TÉCNICO-ECONÓMICOS OBTENIDOS A PARTIR DE LA SIMULACIÓN DEL CASO BASE

EN EL SIMULADOR SUPERPRO DESIGNER ${ }^{\circ}$

Indicador

Inversión total de capital [PC \$]

Costo de operación anual [PC \$/año]

Costo unitario de producción [PC \$/bolsa]

Período de Recuperación de la Inversión (PRI) [años]

Tasa Interna de Retorno (TIR) [\%]

Valor Actual Neto (VAN) [PC \$]

Valor

Costo Total Directo de la Planta (CTDP)

Costo de adquisición del equipamiento [PC \$]

Instalación [PC \$]

Tuberías [PC \$]

Instrumentación [PC \$]

Aislamiento [PC \$]

Instalaciones eléctricas [PC \$]

Edificaciones [PC \$]

Mejoras del terreno [PC \$]

Servicios auxiliares [PC \$]

CTDP [\$]

2.772 .000

3.095 .000

13,05

2,70

33,83

3.842 .000

Costo Total Indirecto de la Planta (CTIP)

Ingeniería [PC \$]

Construcción [PC \$]

Pagos al contratista [PC \$]

Contingencias [PC \$]

CTIP [\$]

Capital Fijo Directo $(C F D)=C T D P+C T I P$ [\$]

Otros

Capital de trabajo [PC \$]

Costo de arrancada [PC \$]

Producción anual de bolsas de leche de cabra en polvo [bolsas/año]

Ingresos anuales gruesos [PC \$/año]

Ingresos anuales netos [PC \$/año]

Ganancias totales anuales [PC \$/año]

Margen grueso [\%]

Retorno de la Inversión [\%]

Tiempo del lote $[\mathrm{h}]$

Número de lotes por año [lotes/año]

$\begin{array}{r}325.000 \\ \hline 455.000 \\ \hline 104.000 \\ \hline 208.000 \\ \hline 1.092 .000 \\ \hline 2.395 .000\end{array}$


Según lo mostrado en la Tabla 5, la inversión total de capital ascendió a \$2,772 millones, con un costo unitario de producción de PC $\$ 13,05 /$ bolsa, obteniéndose un VAN positivo (PC \$3.842.000), una TIR superior al $30 \%(33,83 \%)$ y un PRI inferior a 3 años (2,70 años), lo cual es un indicador de rentabilidad y viabilidad económica (Peters et al., 2003; Baca, 2005; Towler \& Sinnott, 2008; Baca, 2010).

\section{Resultados del estudio de sensibilidad}

La Tabla 7 muestra los resultados del estudio de sensibilidad efectuado tomando en cuenta las 12 corridas realizadas.

TABLA 7. RESULTADOS OBTENIDOS DURANTE EL ESTUDIO
DE SENSIBILIDAD REALIZADO
\begin{tabular}{c|r|r|r}
\hline Corrida & VAN (PC \$) & TIR (\%) & PRI (años) \\
\hline 1 & -10.285 .633 & - & - \\
\hline 2 & -4.140 .680 & - & 1,41 \\
\hline 3 & 11.445 .330 & 64,14 & 9,25 \\
\hline 4 & -1.135 .535 & 4,3 & 2,37 \\
\hline 5 & 5.164 .639 & 36,64 & 3,13 \\
\hline 6 & 3.505 .067 & 26,88 & - \\
\hline 7 & -7.386 .268 & - & 5,12 \\
\hline 8 & 653.034 & 14,3 & 16,36 \\
\hline 9 & -2.298 .748 & - & 5,59 \\
\hline 10 & 328.744 & 12,73 & 1,96 \\
\hline 11 & 7.481 .092 & 45,08 & 1,72 \\
\hline 12 & 8.414 .933 & 51,8 & \\
\hline
\end{tabular}

En la tabla anterior se puede observar que la corrida No. 3 presentó el escenario más optimista tomando en cuenta los valores más elevados obtenidos para los parámetros VAN (PC \$11.445.330) y TIR (64,14\%), asi como el resultado más bajo para el PRI (1,41 años). Lo anterior se debe a que esa corrida tuvo la mayor la mayor capacidad de producción por lote $(600,4 \mathrm{~kg} /$ lote), el menor costo de compra de la leche de cabra fresca (PC \$ $3,5 / \mathrm{L}$ ) y el mayor valor del precio de venta de la bolsa de leche de cabra en polvo (\$23,4/bolsa). Por otra parte, la corrida 1 tuvo el peor escenario económico con un valor de VAN de PC - \$10 285 633, y sin presentar valores de TIR y PRI. Esto se debe a que esta corrida presenta la menor capacidad de producción por lote (355,6 kg/lote), el mayor costo de adquisición de la leche fresca (PC \$ $6,5 / \mathrm{L}$ ) y el menor precio de venta de la bolsa de leche en polvo (PC \$12,6/bolsa).

Resultados obtenidos con respecto a la correlación estadística existente entre las 3 variables de entrada y los indicadores VAN, TIR y PRI

Al efectuar la evaluación de la correlación estadística existente entre los indicadores económicos VAN; TIR y PRI con las tres variables de entrada consideradas, se obtuvo una correlación multi-factorial para cada uno de ellos, las cuales se muestran a continuación.

- Valor Actual Neto

$$
\begin{aligned}
& V A N=-823390-2,78574 \cdot 10^{6 *} C L F+852126 \\
& \text { * PLP+259,549* CLP } \\
& T I R=8,22119-12,1066 * C L F+3,62545 * P L P \\
& +0,00357403 * C L P \\
& P R I=4,90394+2,33866 * C L F-0,694626 * P L P \\
& +0,00129482 * C L P
\end{aligned}
$$

Donde:

CLF - Costo de la leche de cabra fresca

PLP - Precio de la leche de cabra en polvo

CLP - Capacidad de producción de leche en polvo

La Figura 3 muestra los gráficos estadísticos obtenidos mediante el paquete Statgraphics Centurion ${ }^{\circledR}$, relacionados con los datos observados y los predichos para cada una de las tres variables económicas consideradas.

\section{Principales indicadores técnico econó-} mícos obtenidos durante la símulación de la

\section{Variante}

En la Tabla 8 se exponen los resultados de los principales indicadores técnico- económicos obtenidos a partir de la simulación de la Variante en el simulador SuperPro Designer ${ }^{\circledR}$. 
Figura 3. Comparación estadística entre los valores predichos y observados para cada una de las variables económicas consideradas en el estudio

A. Valor Actual Neto (VAN)

B. Tasa Interna de Retorno (TIR)

C. Período de Recuperación de la Inversión (PRI)

A.

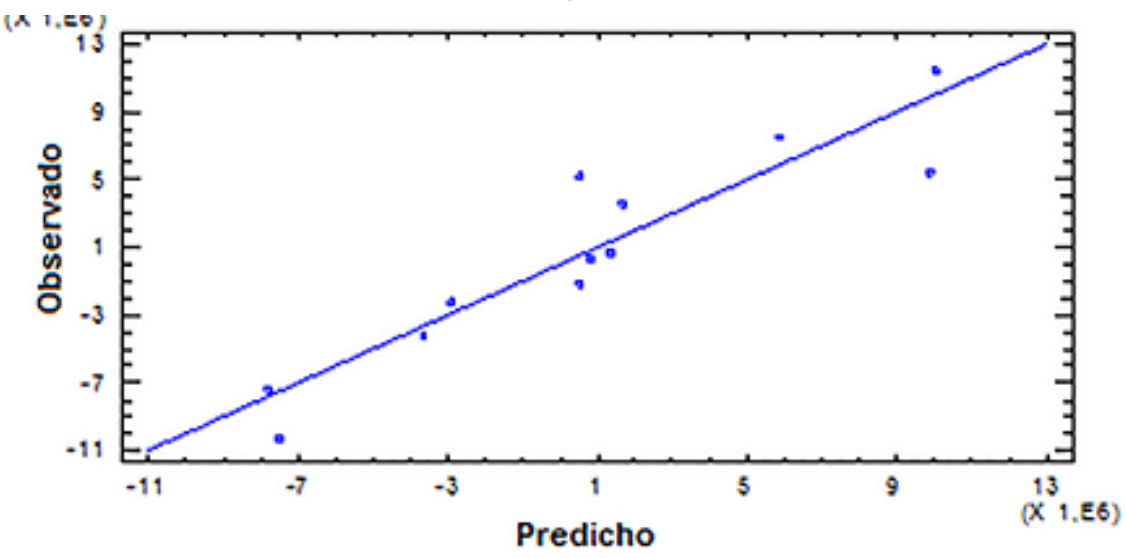

B.

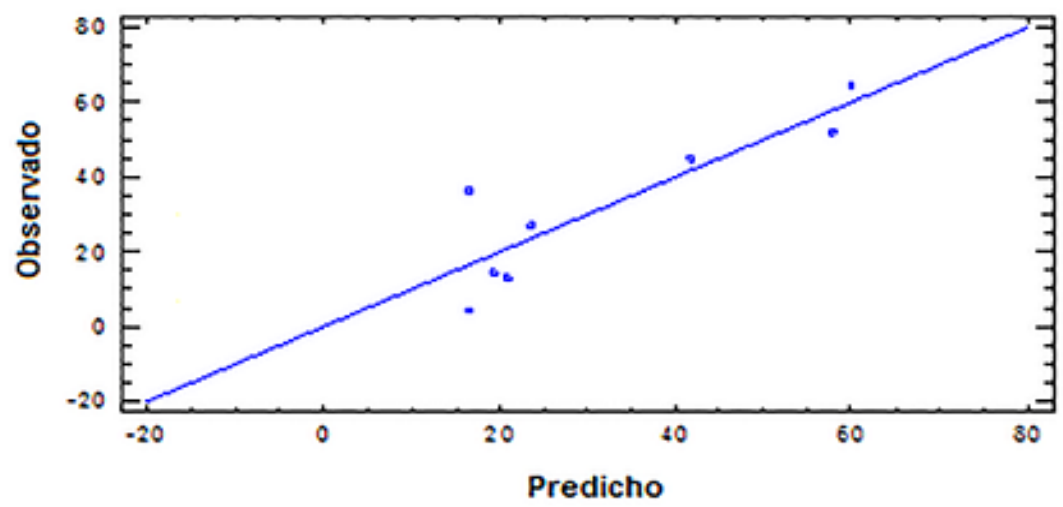

c.

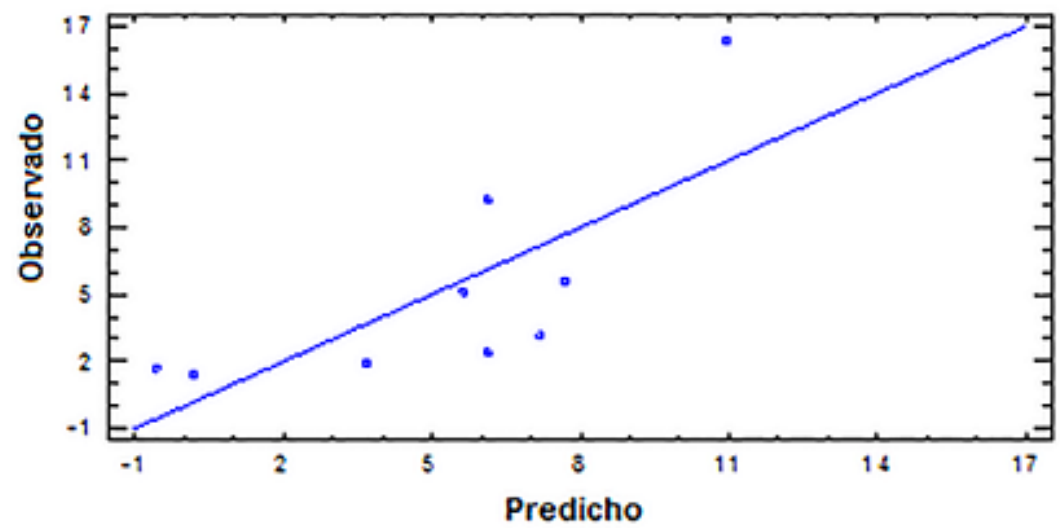




\begin{tabular}{|c|c|}
\hline Indicador & Valor \\
\hline Inversión total de capital [PC \$] & 5.184 .000 \\
\hline Costo de operación anual [PC \$/año] & 5.521 .000 \\
\hline Costo unitario de producción [PC $\$ /$ bolsa] & 14,87 \\
\hline $\begin{array}{l}\text { Período de Recuperación de la Inversión } \\
\text { (PRI) [años] }\end{array}$ & 4,26 \\
\hline Tasa Interna de Retorno (TIR) [\%] & 18,20 \\
\hline Valor Actual Neto (VAN) [PC \$] & 2.030 .000 \\
\hline Capital de trabajo [PC \$] & 460.000 \\
\hline Costo de arrancada [PC \$] & 225.000 \\
\hline $\begin{array}{l}\text { Producción anual de bolsas de leche de } \\
\text { cabra en polvo [bolsas/año] }\end{array}$ & 371.218 \\
\hline Ingresos anuales gruesos [PC \$/año] & 1.161 .000 \\
\hline Ingresos anuales netos [PC \$/año] & 1.217 .000 \\
\hline Ganancias totales anuales [PC \$/año] & 6.682 .000 \\
\hline Margen grueso [\%] & 17,38 \\
\hline Retorno de la Inversión [\%] & 23,47 \\
\hline Tiempo del lote $[\mathrm{h}]$ & 21,63 \\
\hline Número de lotes por año [lotes/año] & 487 \\
\hline
\end{tabular}

La Figura 4 muestra una comparación de los valores de VAN, TIR, PRI, costo unitario de producción, ingresos anuales netos, margen grueso, retorno de la inversión e inversión total de capital obtenidos para la Variante con respecto al Caso Base.

\section{Figura 4. Comparación de los principales indicadores téc-} nico-económicos obtenidos para el Caso Base y la Variante

A. VAN
B. TIR
C. PRI
D. Costo unitario de
producción

E. Ganancias netas anuales F. Margen grueso

G. Retorno de la inversión H. Inversión total de capital A.

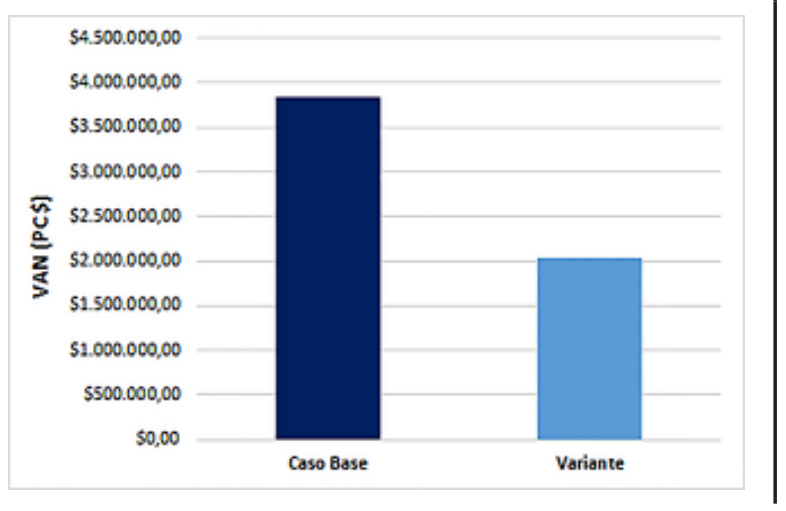

B.

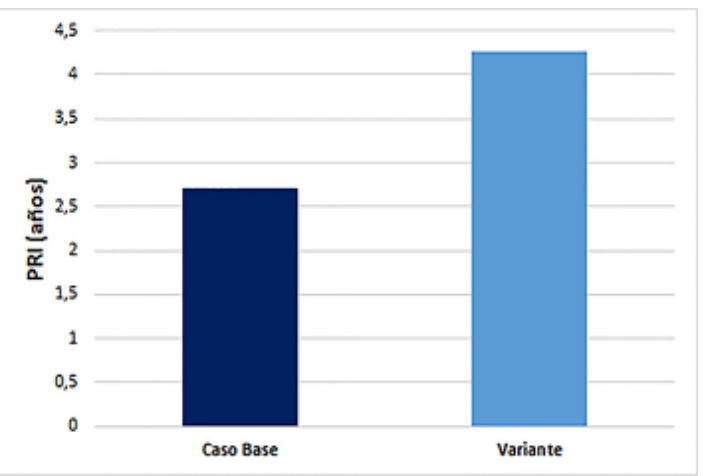

C.

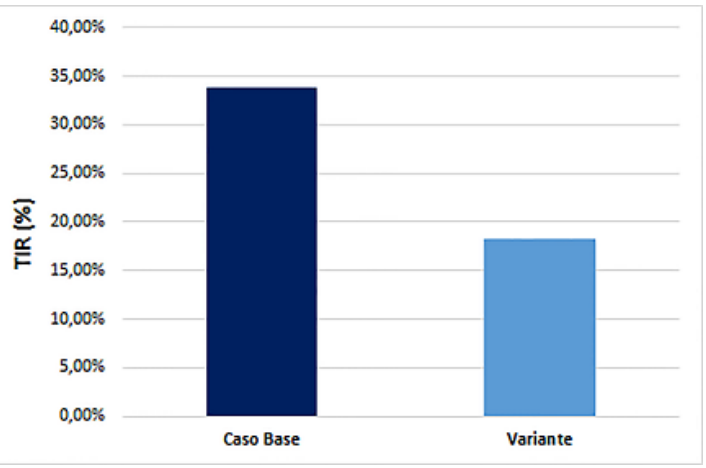

D.

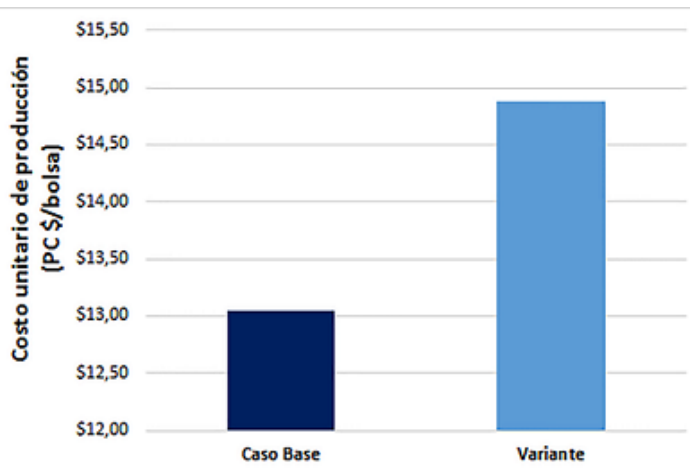

E.

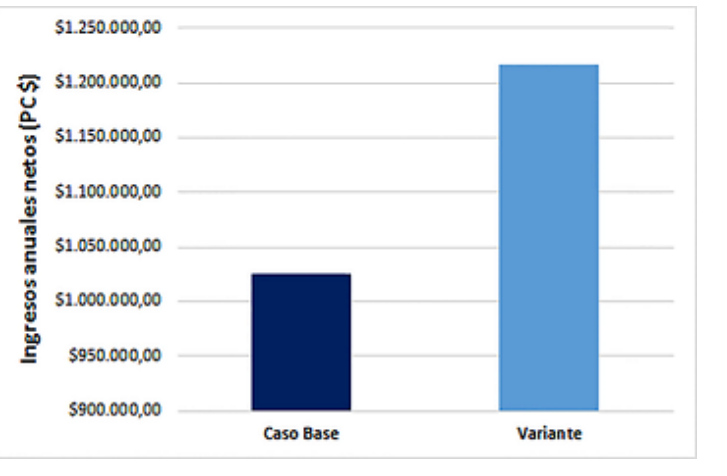




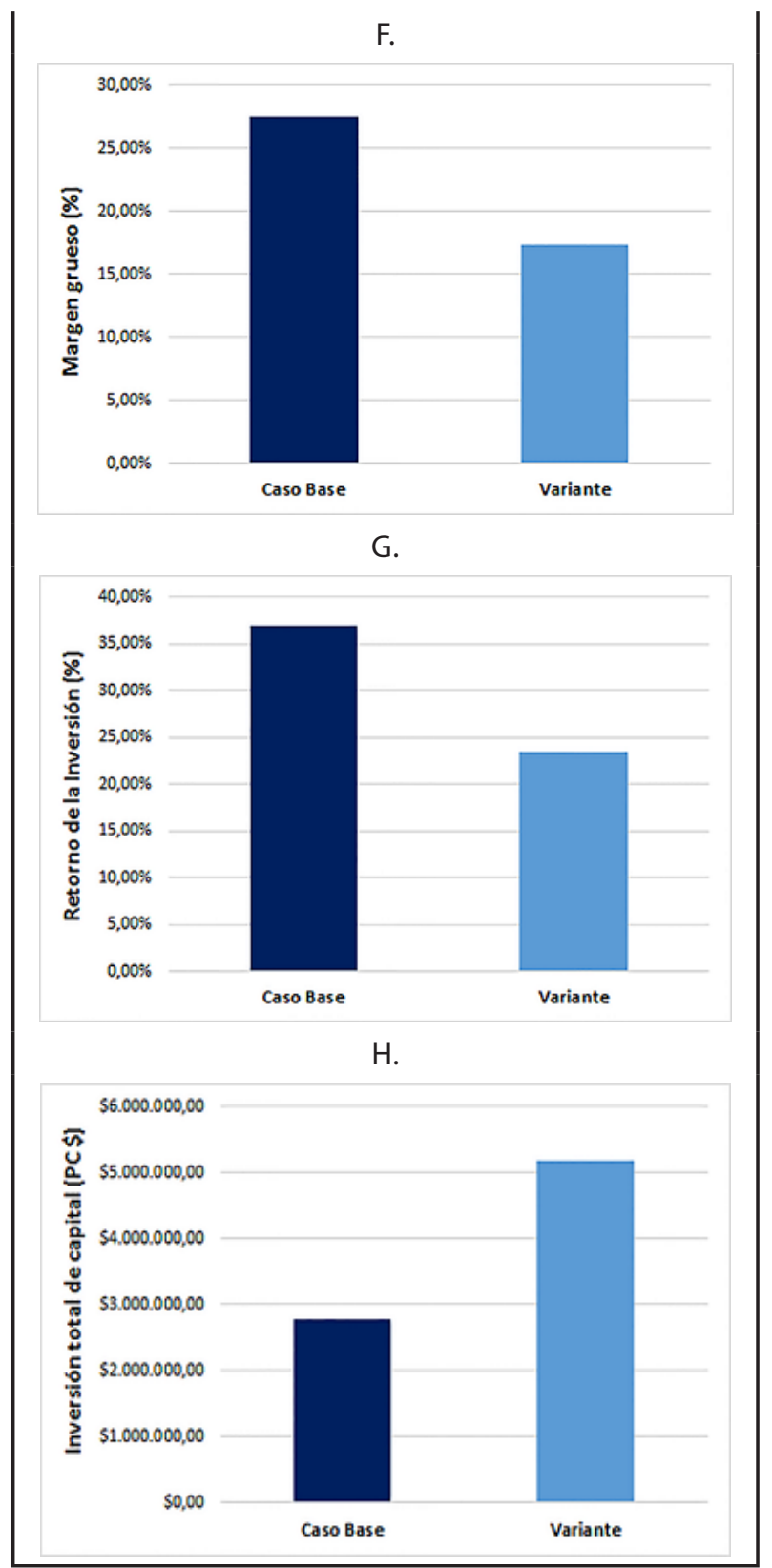

De acuerdo con los resultados mostrados en la Tabla 7, la introducción de un segundo secador por aspersión en el proceso productivo reduce el valor del VAN en 1,89 veces y el valor de la TIR en 1,86 veces, mientras que incrementa el valor del PRI en 1,58 veces para la Variante con respecto al Caso Base. También se incrementa la inversión total de capital en un 46,5\%, los costos anuales de operación en un $43,9 \%$, el costo unitario de la bolsa de leche en polvo en un $12,2 \%$, la producción anual de bolsas en un $36,1 \%$, el número total de lotes anuales en un 42,5 \% y las ganancias totales anuales en un $36,1 \%$. Por otra parte se reducen el margen grueso en un $36,7 \%$, el retorno de la inversión en un $36,5 \%$ y el tiempo de un lote en un $35,7 \%$. Por último, se incrementa tanto el capital de trabajo como el costo de arrancada en PC \$ 201000 y PC \$ 105 000, respectivamente. Esto es, se incrementan los costos fijos, los costos de operación, el costo unitario de producción, así como también las ganancias anuales a obtener, mientras que se reducen los valores de VAN, TIR, retorno de la inversión y el margen grueso del proceso. Los resultados obtenidos indican que no resulta rentable incluir un secador por aspersión adicional en el proceso productivo debido a que reduce los resultados de VAN y TIR, mientras que incrementa el valor del PRI en comparación con los valores obtenidos de estos parámetros en el Caso Base, disminuyendo además otros indicadores económicos de importancia tales como el retorno de la inversión y el margen grueso.

\section{Conclusiones}

1. La simulación del Caso Base permitió obtener valores de VAN, TIR y PRI de PC \$ 3842 000, 33,83\% y 2,70 años, respectivamente, calificando el proceso productivo de económicamente rentable.

2. La simulación de la Variante condujo a la obtención de valores de VAN, TIR y PRI de PC \$2.030.000, $18,20 \%$ y 4,26 años, respectivamente.

3. La introducción de un secador por aspersión adicional (Variante) en el proceso productivo disminuye la rentabilidad global del mismo, ya que disminuyen los valores de VAN y TIR en 1,89 y 1,86 veces, respectivamente, y se incrementa el valor del PRI en 1,58 veces.

4. Se obtuvieron correlaciones estadísticas que relacionan los indicadores VAN, TIR y PRI con el costo de la leche fresca, el precio de la leche en polvo y la capacidad de producción de la leche en polvo.

\section{Referencias}

Altieri, G.; Renzo, G. C. D.; Genovese, F. (2008). Preliminary results using a new method to optimize a spray dryer process for producing high quality milk powder from cow, goat and she-ass milk concentrates. J. of Ag. Eng., 4, pp. 35-41. https://doi.org/10.4081/jae.2008.4.35 
Auli, N. A.; Sakinah, M.; Bakri, A. M. M. A.; Kamarudin, H.; Norazian, M. N. (2013). Simulation Of Xylitol Production: A Review. Australian Journal of Basic and Applied Sciences, 7(5), pp. 366-372.

Baca, G. (2005). Ingenieria Económica (8va ed.). Bogotá D.C, Fondo Educativo Panamericano, pp. 112-159.

Baca, G. (2010). Evaluación de proyectos. México D.F., McGraw-Hill/Interamericana Editores S.A de C.V, pp. 56-94.

Correia, R. T. P.; Magalhães, M. M. d. A.; Pedrini, M. R. d. S.; Cruz, A. V. F. d.; Clementino, I. (2008). Ice cream made from cow and goat milk: chemical composition and melting characteristics. Revista Ciencia Agronomica, 39(2), pp. 251-256. https://doi.org/10.12691/ jfnr-1-4-6

Domagala, J. (2009). Instrumental texture, syneresis and microstructure of yoghurts prepared from goat, cow and sheep milk. Int. J. Food Prod., 12, pp. 605-615. https://doi.org/10.1080/10942910801992934

Fox, P. F., McSweeney, P. L. H. (1998). Dairy Chemistry and Biochemistry (1st ed.). London: Blackie Academic \& Professional.

Gomes, A. M. P.; Malcata, F. X. (1998). Development of probiotic cheese manufactured from goat milk: response surface analyses via technological manufacture. J. Dairy Sci., 81, pp. 1492-1507. https://doi.org/10.3168/ jds.S0022-0302(98)75715-7

Guo, M.; Park, Y. W.; Dixon, P. H.; Gilmore, J. A.; Kindstedt, P. S. (2004). Relationship between the yield of cheese (Chevre) and chemical composition of goat milk. Small Ruminant Research, 52, pp. 103-107. https://doi. org/10.1016/S0921-4488(03)00247-5

Kücükcetin, A.; Demir, M.; Ascı, A.; Comak, E. M. (2011). Graininess and roughness of stirred yoghurt made with goat's, cow's or a mixture of goat's and cow's milk. Small Ruminant Research, 96, pp. 173-177. https://doi. org/ 10.1016/j.smallrumres.2010.12.003

Lam, K. F.; Leung, C. C. J.; Lei, H. M.; Lin, C. S. K. (2014). Economic feasibility of a pilot-scale fermentative succinic acid production from bakery wastes. Food and Bioproducts Processing, 92, pp. 282-290. https://doi. org/10.1016/j.fbp.2013.09.001

Mukdsi, M. C. A.; Haroc, C.; Gonzȧlez, S. N.; Medina, R. B. (2013). Functional goat milk cheese with feruloyl esterase activity. Journal of Functional Foods, 5, pp. 801-809. https://doi.org/10.1016/j.jff.2013.01.026

Pal, M. (2014). Goat milk and its potential in dairy industry, (MSc Thesis), Debre Zeit, Ethiopia, Addis Ababa University, College of Veterinary Medicine, $116 \mathrm{pp}$.
Pal, M.; Dudhrejiya, T. P.; Pinto, S. (2017). Goat Milk Products and their significance. Beverage \& Food World, 44(7), pp. 21-25.

Pandya, A. J.; Ghodke, K. M. (2007). Goat and sheep milk products other than cheeses and yoghurt. Small Ruminant Research, 68, pp. 193-206. https://doi.org/10.1016/j. smallrumres.2006.09.007

Park, Y.W. (1990). Nutrient profiles of commercial goat milk cheeses manufactured in the United States. J. Dairy Sci., 73, pp. 3059-3067. https://doi.org/10.3168/jds. S0022-0302(90)78993-X

Perry, R. H., Green, D. (2008). Chemical Engineers' Handbook. New York, McGraw-Hill, pp. 9-1 - 9.56.

Peters, M., Timmerhaus, K., West, R. (2003). Plant design and Economics for Chemical Engineers. Boston, McGraw-Hill, pp. 226-275.

Prosser, C. G.; McLaren, R. D.; Frost, D.; Agnew, M.; Lowry, D. J. (2008). Composition of the non-protein nitrogen fraction of goat whole milk powder and goat milkbased infant and follow-on formulae. International Journal of Food Sciences and Nutrition, 59, pp. 123-133. https://doi.org/10.1080/ 09637480701425585

Qureshi, N.; Saha, B. C.; Cotta, M. A.; Singh, V. (2013). An economic evaluation of biological conversion of wheat straw to butanol: A biofuel. Energy Conversion and Management, 65, pp. 456-462. https://doi.org/10.1016/j. enconman.2012.09.015

Ranadheera, C. S.; Evans, C. A.; Adams, M. C.; Baines, S. K. (2013). Production of probiotic ice cream from goat's milk and effect of packaging materials on product quality. Small Ruminant Research, 112, pp. 174-180. https://doi.org/10.1016/ j.smallrumres.2012.12.020

Ravula, S. R.; Ramachandra, C.; Ravula, P. R.; Hiregoudar, S.; Nidoni, U. K. (2018). Sensory evaluation of spray dried and agglomerated goat milk powder and their comparison. The Pharma Innovation Journal, 7(7), pp. 835-838.

Reddy, R. S.; Ramachandra, C. T.; Hiregoudar, S.; Nidoni, U.; Ram, J.; Kammar, M. (2014). Influence of processing conditions on functional and reconstitution properties of milk powder made from Osmanabadi goat milk by spray drying. Small Ruminant Research, 119, pp. 130-137. https://doi.org/10.1016/ j.smallrumres.2014.01.013

Rodriguez, A.; Bunger, A.; Castro, E.; Sousa, I.; Empis, J. (2003). Development and optimization of cultured goat cream butter. Journal of American Oil Chemical Society, 80, pp. 987-992. https://doi.org/10.1007/ s11746-003-0808-8 
Seifu, E.; Buys, E. M.; Donkin, E. F. (2004). Quality aspects of Gouda cheese made from goat milk preserved by the lactoperoxidase system. International Dairy Journal, 14, pp. 581-589. https://doi.org/10.1016/j. idairyj.2003.12.006

Towler, G., Sinnott, R. (2008). Chemical Engineering Design - Principles, Practice and Economics of Plant and Process Design. London, Butterworth-Heinemann, pp. 297-392.

Vargas, M.; Chafer, M.; Albors, A.; Chiralt, A.; GonzalezMartinez, C. (2008). Physicochemical and sensory characteristics of yoghurt produced from mixtures of cows' and goats' milk. International Dairy Journal, 18, pp. 1146-1152. https://doi.org/10.1016/j. idairyj.2008.06.007

Yangilar, F. (2013). As a Potentially Functional Food: Goats' Milk and Products. Journal of Food and Nutrition Research, 1(4), pp. 68-81. https://doi.org/10.12691/ jfnr-1-4-6
PARA CITAR ESTE ARTÍCULO TO REFERENCE THIS ARTICLE / PARA CITAR ESTE ARTIGO /

Crespo-Zafra, L.; Pérez-Sánchez, A.; Ramos-López, L.; García-Noa, E.; Matos-Mosqueda, L. (2020). Estudio técnico-económico de la producción de leche de cabra en polvo. Revista EIA, 17(33) enero-junio, Reia33008 pág. 1-13. Disponible en: https://doi.org/10.24050/reia.v17i33.1330 